\title{
Using the Markov Chain Monte Carlo method to make inferences on items of data contaminated by missing values
}

\author{
I. Karangwa, D. Kotze \\ Department of Statistics and Population Studies, University of the Western Cape, Cape Town, South Africa
}

Email address:

fokafoka15@gmail.com (I. Karangwa),dkotze@uwc.ac.za (D. Kotze)

\section{To cite this article:}

I. Karangwa, D. Kotze. Using the Markov Chain Monte Carlo Method to Make Inferences on Items of Data Contaminated by Missing Values, American Journal of Theoretical and Applied Statistics. Vol. 2, No. 3, 2013, pp.48-53. doi: 10.11648/j.ajtas.20130203.12

\begin{abstract}
The Markov Chain Monte Carlo (MCMC) is a method that is used to estimate parameters of interest under difficult conditions such as missing data or when underlying distributions do not fit the assumptions of Maximum Likelihood processes. The objective of this process is to find a probability distribution known as a posterior distribution in Bayesian analysis that can be used to estimate target parameters. In this paper, we consider a case where data are contaminated with missing values and therefore need to be adequately handled using missing data techniques before making inferences on them. A review of the mathematics involved in MCMC procedures in the presence of missing data is presented. Furthermore, we use real data to compare inferences made using multiple imputation based on the multivariate normal model (MVN) that uses the MCMC procedure, the case deletion (CD) missing data method that discards subjects with missing values from the analysis, and the fully conditional specification (FCS) multiple imputation method that uses a sequence of regression models to fill in missing values. Assuming that data are missing completely at random (MCAR) on continuous and normally distributed variables, the following findings are obtained: (1) The higher the proportion of missing data on a variable of interest, the more the relationship between that variable and the dependent variable is distorted when all missing data methods are applied. (2) Multiple imputation based methods produce similar estimates which are better than estimates from the case deletion method. (3) At some stage (when the proportion of missing data becomes high), none of the missing data techniques can help to maintain an initially existing relationship between the dependent variable and some of the covariates of interest in the dataset.
\end{abstract}

Keywords: Markov Chain Monte Carlo (MCMC), Missing Data, Missing Completely At Random (MCAR), Multiple Imputation, Multivariate Normal Model (MVN), Fully Conditional Specification (FCS)

\section{Introduction}

Missing data are common and a major problem in different fields of research $[6,9,16]$. It is not given much attention by some researchers especially those who are not methodologists or statistical experts. This is due mainly to the lack of familiarity with the existing statistical literature on missing values or the ignorance of the impact that missing data can have on statistical inferences [13]. A traditional way of dealing with missing data is to eliminate these observations from the analysis, a strategy that is referred to as the Case Deletion or Listwise Deletion (CD) method. Discarding missing observations from analysis reduces the sample size, which leads to a sample that is not representative of the population. Consequently, the power of the sta- tistical test is reduced and biased parameter estimates and large standard errors are obtained, especially when a large amount of data are missing $[2,11,13]$. To avoid some of these problems, researchers have come up with ways of rescuing missing data in order to make valid inferences [13, $15,16]$. These include weighting (to reduce the effect of attributes with a large percentage of missing values), modelling using methods such as the maximum likelihood (ML) estimation and available data to recover missing data, or imputing missing data using certain means of approximation based on observed data, for instance the mean imputation, hot-deck imputation, regression imputation, multiple imputation using the Markov Chain Monte Carlo method, etc. The primary goal of these methods is to obtain valid and efficient statistical inferences about the population of interest, but not to recover missing data or to find what 
would have been obtained if we had complete data [14] This paper focuses mainly on the data augmentation method based on a multivariate normal model (MVN) that uses the Markov Chain Monte Carlo procedure. It assumes a normal distribution for the variables in the imputation model [14].

A number of studies compared this method to the fully conditional specification (FCS) method which is also a multiple imputation-based method that uses a sequence of regression models to impute missing values, depending on the nature of the variables to be imputed; linear regression for continuous variables, logistic regression for binary variables, multinomial regression for polytomous variables, etc. $[10,17,19]$. These studies concentrated on different aspects and applied the two methods to data with a mixture of variables (continuous, discrete and semi-continuous). Mixed results were obtained; some concluded that the MVN was better than the FCS [17, 19] and others found the opposite [1, 10]. Van Buuren [17] also highlighted that the two methods are identical when applied to continuous and normally distributed data. In this paper, we use different simulated data sets to also give our view of these two methods in terms of their performance when data are missing completely at random (MCAR) on continuous and normally distributed variables.

\section{Markov Chain Monte Carlo Methods in the Presence of Missing Values in a Dataset}

When data are missing, the primary objective of a researcher is to generate unbiased estimates in order to make good inferences. This is not generally easy when available data (observed data after discarding missing items) are used. With MCMC methods, the available data need to be augmented with simulated values of the missing data in order to get good parameter estimates. The procedure is as follows.

Let $\mathrm{Y}$ be multivariate data from a normal distribution, $p(Y \mid \theta)=N(\beta, \Sigma)$, where $\theta$ denotes the unknown model parameters such as regression coefficients $\beta$ and covariance matrix $\Sigma$. In the presence of missing values we have $Y=\left(Y_{o b s}, Y_{m i s}\right)$, where $Y_{o b s}$ and $Y_{m i s}$ represent the observed and missing parts respectively. Missing values in $Y_{m i s}$ are drawn from the distribution of missing data given the observed data, $p\left(Y_{m i s} \mid Y_{o b s}\right)$. However, this distribution is difficult to sample from directly because it depends on the posterior distribution of the unknown parameters $\theta$, $p\left(\theta \mid Y_{o b s}\right)$. Initially, the data augmentation (DA) method was designed to approximate this distribution when data are incomplete [14]. The observed data need to be augmented with unobserved data $Y_{m i s}$ such that the conditional distribution $p\left(Y_{m i s} \mid Y_{o b s}\right)$ becomes easier to sample from. To illustrate the above, consider the posterior distribution $p\left(\theta \mid Y_{o b s}\right)$. When data are incomplete, this distribution can be written in terms of an integral as follows:

$$
p\left(\theta \mid Y_{o b s}\right)=\int p\left(\theta \mid Y_{o b s}, Y_{m i s}\right) p\left(Y_{m i s} \mid Y_{o b s}\right) d Y_{m i s}
$$

To evaluate this density, the Monte Carlo method can be used. That is independent copies of Ymis(Ymis(1),Ymis(2), ..., Ymis(n)) can be drawn from the conditional distribution $\mathrm{p}$ (Ymis | Yobs) and then the average $\frac{1}{n} \sum p(\theta \mid Y(j))$ can be computed and serve as an approximation of $\mathrm{p}$ (Yobs), where $\mathrm{Y}(\mathrm{j})$ denotes the augmented dataset (Yobs,Ymis,(j)) for $\mathrm{j}=1,2, \ldots, \mathrm{n}$ and $\operatorname{Ymis}(\mathrm{j})=(\mathrm{Ymis}) 1(\mathrm{j})$, (Ymis)2(j), ..., (Ymis)n(j).

In the Markov Chain Monte Carlo context, the above mentioned idea can be simply implemented using the Imputation-Parameter (IP) algorithm suggested by Schafer [14] which works as follows. Assuming a multivariate normally distributed data, at the th iteration one needs to draw $Y_{m i s}^{(t+1)}$ from $p\left(Y_{m i s} \mid Y_{o b s}, \theta^{(t)}\right)$, and then draw $\theta^{(t+1)}$ from $p\left(\theta \mid Y_{o b s}, Y_{m i s}^{(t+1)}\right)$. The former step is referred to as the Imputation (I) step and the latter as the Parameter (P) step. The resulting sequence forms a Markov chain $\left\{Y_{m i s}^{(1)}, \theta^{(1)}\right\},\left\{Y_{m i s}^{(2)}, \theta^{(2)}\right\}, \ldots,\left\{Y_{m i s}^{(t+1)}, \theta^{(t+1)}\right\}$ which must converge to the distribution $p\left(Y_{m i s} \mid Y_{o b s}, \theta\right)[3,7,8]$ and used in the Multiple Imputation to estimate parameters when data are missing. In words, in the I-step missing values $Y_{m i s}$ are simulated for each observation independently by using the observed data Yobs and the estimates of the mean vector and covariance matrix represented by $\theta^{(t)}$. The P-step uses the complete data set (full data with generated missing values) from the I-step to generate new estimates of the mean vector and covariance matrix, which are to be used in the next I-step to simulate new values. The repetition of these two steps (I-step and P-step) creates a Markov chain (sequence of random variables in which the distribution of each element is related to the values of the previous one). Their role is to generate a distribution of values from which random samples of simulated missing values are obtained and used in Multiple Imputation methods to estimate parameters when data are missing. The chain needs to be long enough for the distribution of the elements to stabilize to a common distribution referred to as the stationary distribution [14].

\section{Methodology}

\subsection{Description of the Original Data Set}

Data from the Demographic and Health Surveys (DHSs) conducted in the Democratic Republic of Congo (DRC) in 2007 was used for the analysis. It consists of a household and women's questionnaire where a nationally representative sample of women between 15 and 49 years of age were interviewed regardless of marital status in each sampled household. Information was collected on fertility and family planning in addition to socio-demographic and eco- 
nomic data. The sample of women in the analysis includes women of reproductive age who were not pregnant at the time of interview and who were sexually active. Respondents were asked about their knowledge and use of contraception methods, etc. Information on whether they have ever used contraception was first obtained and then the types of contraception methods used were asked. Contraception methods used included the modern (i.e. pill, injections and other), traditional (i.e. abstinence and other) and folkloric (i.e. herbal plant and other) methods. The dependent variable considered for the analysis was the women's contraceptive use status measured as any contraceptive method use by including all women who reported using modern, traditional and folkloric methods coded as 1 and 0 to represent women who have never used any contraceptive method. The purpose was to determine whether and to what extent certain covariates such as the age and education in years of a woman, her marital status, etc., are associated with the woman's use of contraception. Thus, slopes, standard errors and results of hypothesis tests were considered as outcomes of interest to be analyzed.

\subsection{Simulation of Data Sets with Missing Values}

In order to assess the performance of the Multiple Imputation using the Markov Chain Monte Carlo method or MVN and other methods (case deletion and fully conditional specification), datasets were created with some of their values missing completely at random (MCAR) on variables age and education that were statistically significant in the regression model. This assumption means that missingness probabilities are not related at all to any other variables in the data set.

As an application, eight data sets were created with different rates of missingness; $5 \%, 10 \%, 15 \%, 20 \%, 25 \%, 30 \%$, $35 \%$ and $40 \%$ on variables age and education. Based on the variables of interest (age and education) with no missing data, a 0 - 1 random generator if the observation was missing (1) or not (0) was constructed. This means that missing data are random draws from the Bernouilli distribution with the parameter $p$ that represents the percentage of missing values of interest (percentages of missingness in this case). Technically, this can be represented as follows. Let $\mathrm{Y}_{\mathrm{i}}$ be a complete data vector for respondent $i$. Then $Y_{i}$ can be partitioned into $\mathrm{Y}_{\mathrm{i}, \text { obs }}$ and $\mathrm{Y}_{\mathrm{i}, \mathrm{mis}}$, the observed and missing parts respectively. That is, $Y_{i}=\left(Y_{i, o b s}, Y_{i, m i s}\right)$. Let also $R_{i}=\left(r_{i j}\right)$ be the missing data indicator, where $r_{i j}=1$ if a value is missing and $\mathrm{r}_{\mathrm{ij}}=0$ otherwise. Given some parameter $\boldsymbol{\theta}$, the MCAR assumption states that

$$
p\left(r_{r j} \mid Y_{i, o b s}, Y_{i, m i s}, \theta_{i}\right)=p\left(r_{i j}, \theta_{i}\right)
$$

This means that the distribution of missingness does not depend on the data at all and can be seen as a Bernouilli distribution with the probability density function

$$
\prod p\left(r_{r j} \mid \theta_{r j}\right)=\theta_{j}^{r j}\left(1-\theta_{j}\right)^{1-r j}=p_{i}^{r j} q^{1-r j}
$$

$$
\text { for } q=1-p_{i}^{r j} \text { and } r j \in(0.1) \text {. }
$$

The $\mathrm{R}$ and STATA 12 statistical software packages were utilized for the analysis. The former was used to simulate datasets with missing values and the latter was employed to fit different regression models.

\subsection{Analysis Method}

For each simulated data set with missing values on the variables of interest (age and education in years), the multiple imputation method which assumes the multivariate normal model (MVN) and the fully conditional specification (FCS) that use a sequence of regression models to impute missing values, were applied. Then regression models were estimated on the data set with no missing values (original data), data sets with missing values (incomplete data), as well as on the imputed data sets. The results were compared in terms of slopes, standard errors and p-values. The MVN method was performed using Stata implementation of Schafer's NORM program [4] whereas the FCS was carried out using the mice command in Stata [18].

\section{Results}

Table 1 presents the results of the parameters' estimation, standard errors and p-values of the two slopes from the binary regression of women's contraceptive use status (dependent variable) on their age and education in years (independent variables). The datasets considered in the analysis were first the original data with age and education without missing values. Secondly, 8 datasets were employed with $5 \%, 10 \%, 15 \%, 20 \%, 25 \%, 30 \%, 35 \%$ and $40 \%$ of the information missing completely at random on these two variables. The results indicated that multiple imputation-based methods (MVN and FCS) produce less biased estimates than the case deletion method. It was also shown that the MVN and FCS yield similar parameter estimates (see Table 1, Figure 1 and Figure 2) when applied to continuous and normally distributed variables. Furthermore, we also found that all the missing data methods considered in the analysis overestimate the standard errors of the models (Figure 3 and Figure 4). In Figure 3 and 4, we observe that the $\mathrm{CD}$ biases standard errors more than the MVN and the FCS, and the larger the percentage of missing data, the more inflated standard errors are obtained. In Figure 5 we see that at lower percentages of missing values (at most $15 \%$ ), the three missing data methods produce similar and unbiased p-values, otherwise the CD yields higher $\mathrm{p}$-values. In addition, the findings indicated that the higher the per centage of missing data, the more the relationship between the dependent and the independent variables (which were all statistically significant in the model with no missing data) is distorted when missing observations are excluded from the analysis. Indeed, the results showed that when at least $20 \%$ of observations are missing on independent variables, some of them lose their statistically significant relationship with the dependent variable. We also observed that 
in general, the data augmentation method using the MCMC procedure produces estimates that are close to the estimates of the fitted model with no missing data as compared to the estimates of the model fitted after discarding missing values. Finally, it was observed that at some stage (when at least $25 \%$ of the data are missing), neither the imputation nor the Case Deletion can help to maintain the relationship that exists between the dependent and independent variables when the analysis is done using the dataset with no missing values (see Table 1).

Table 1. Parameter estimates of a set of logistic regression models for predicting the contraceptive methods use status by women of reproductive age in Democratic Republic of Congo (DRC), using age ( $1^{\text {st }}$ covariate) and education (2 ${ }^{\text {nd }}$ covariate) in years as explanatory variables.

\begin{tabular}{|c|c|c|c|c|c|c|c|c|c|}
\hline Proportion of missing data & $\mathbf{B}_{\mathbf{i}^{* *}}$ & & & $\mathrm{SE}^{* * *}$ & & & $\mathbf{P V} * * * *$ & & \\
\hline & CD & MVN & FCS & CD & MVN & FCS & CD & MVN & FCS \\
\hline 0.00 & $\begin{array}{l}0.0261 \\
0.0067\end{array}$ & $\begin{array}{l}\text { NA } \\
\text { NA }\end{array}$ & $\begin{array}{l}\text { NA } \\
\text { NA }\end{array}$ & $\begin{array}{l}0.0029 \\
0.0026\end{array}$ & $\begin{array}{l}\text { NA } \\
\text { NA }\end{array}$ & $\begin{array}{l}\text { NA } \\
\text { NA }\end{array}$ & $\begin{array}{l}0.000 * \\
0.011 *\end{array}$ & $\begin{array}{l}\text { NA } \\
\text { NA }\end{array}$ & $\begin{array}{l}\text { NA } \\
\text { NA }\end{array}$ \\
\hline 0.05 & $\begin{array}{l}0.0272 \\
0.0067\end{array}$ & $\begin{array}{l}0.0267 \\
0.0069\end{array}$ & $\begin{array}{l}0.0272 \\
0.0073\end{array}$ & $\begin{array}{l}0.0032 \\
0.0028\end{array}$ & $\begin{array}{l}0.0030 \\
0.0027\end{array}$ & $\begin{array}{l}0.0030 \\
0.0027\end{array}$ & $\begin{array}{l}0.000^{*} \\
0.017 *\end{array}$ & $\begin{array}{l}0.000 * \\
0.010 *\end{array}$ & $\begin{array}{l}0.000^{*} \\
0.007 *\end{array}$ \\
\hline 0.10 & $\begin{array}{l}0.0251 \\
0.0065\end{array}$ & $\begin{array}{l}0.0269 \\
0.0067\end{array}$ & $\begin{array}{l}0.0271 \\
0.0071\end{array}$ & $\begin{array}{l}0.0033 \\
0.0029\end{array}$ & $\begin{array}{l}0.0032 \\
0.0027\end{array}$ & $\begin{array}{l}0.0031 \\
0.0028\end{array}$ & $\begin{array}{l}0.000^{*} \\
0.028^{*}\end{array}$ & $\begin{array}{l}0.000 * \\
0.014 *\end{array}$ & $\begin{array}{l}0.000^{*} \\
0.011^{*}\end{array}$ \\
\hline 0.15 & $\begin{array}{l}0.0302 \\
0.0065\end{array}$ & $\begin{array}{l}0.0296 \\
0.0078\end{array}$ & $\begin{array}{l}0.0284 \\
0.0076\end{array}$ & $\begin{array}{l}0.0037 \\
0.0031\end{array}$ & $\begin{array}{l}0.0034 \\
0.0031\end{array}$ & $\begin{array}{l}0.0034 \\
0.0028\end{array}$ & $\begin{array}{l}0.000 * \\
0.021 *\end{array}$ & $\begin{array}{l}0.000 * \\
0.006 *\end{array}$ & $\begin{array}{l}0.000^{*} \\
0.006^{*}\end{array}$ \\
\hline 0.20 & $\begin{array}{l}0.0293 \\
0.0045\end{array}$ & $\begin{array}{l}0.0290 \\
0.0059\end{array}$ & $\begin{array}{l}0.0279 \\
0.0052\end{array}$ & $\begin{array}{l}0.0041 \\
0.0033\end{array}$ & $\begin{array}{l}0.0037 \\
0.0030\end{array}$ & $\begin{array}{l}0.0035 \\
0.0028\end{array}$ & $\begin{array}{l}0.000^{*} \\
0.172\end{array}$ & $\begin{array}{l}0.000 * \\
0.045^{*}\end{array}$ & $\begin{array}{l}0.000 * \\
0.066\end{array}$ \\
\hline 0.25 & $\begin{array}{l}0.0275 \\
0.0027\end{array}$ & $\begin{array}{l}0.0268 \\
0.0045\end{array}$ & $\begin{array}{l}0.0279 \\
0.0038\end{array}$ & $\begin{array}{l}0.0041 \\
0.0035\end{array}$ & $\begin{array}{l}0.0033 \\
0.0028\end{array}$ & $\begin{array}{l}0.0036 \\
0.0031\end{array}$ & $\begin{array}{l}0.000^{*} \\
0.451\end{array}$ & $\begin{array}{l}0.000^{*} \\
0.105\end{array}$ & $\begin{array}{l}0.000^{*} \\
0.075\end{array}$ \\
\hline 0.30 & $\begin{array}{l}0.0252 \\
0.0029\end{array}$ & $\begin{array}{l}0.0263 \\
0.0043\end{array}$ & $\begin{array}{l}0.0262 \\
0.0038\end{array}$ & $\begin{array}{l}0.0041 \\
0.0037\end{array}$ & $\begin{array}{l}0.0038 \\
0.0031\end{array}$ & $\begin{array}{l}0.0037 \\
0.0032\end{array}$ & $\begin{array}{l}0.000^{*} \\
0.433\end{array}$ & $\begin{array}{l}0.000 * \\
0.169\end{array}$ & $\begin{array}{l}0.000 * \\
0.228\end{array}$ \\
\hline 0.35 & $\begin{array}{l}0.0262 \\
0.0023\end{array}$ & $\begin{array}{l}0.0264 \\
0.0043\end{array}$ & $\begin{array}{l}0.0265 \\
0.0041\end{array}$ & $\begin{array}{l}0.0047 \\
0.0040\end{array}$ & $\begin{array}{l}0.0034 \\
0.0032\end{array}$ & $\begin{array}{l}0.0038 \\
0.0032\end{array}$ & $\begin{array}{l}0.000^{*} \\
0.564\end{array}$ & $\begin{array}{l}0.000 * \\
0.177\end{array}$ & $\begin{array}{l}0.000^{*} \\
0.211\end{array}$ \\
\hline 0.40 & $\begin{array}{l}0.0262 \\
0.0008\end{array}$ & $\begin{array}{l}0.0248 \\
0.0047\end{array}$ & $\begin{array}{l}0.0250 \\
0.0043\end{array}$ & $\begin{array}{l}0.0050 \\
0.0044\end{array}$ & $\begin{array}{l}0.0041 \\
0.0033\end{array}$ & $\begin{array}{l}0.0039 \\
0.0037\end{array}$ & $\begin{array}{l}0.000^{*} \\
0.855\end{array}$ & $\begin{array}{l}0.000 * \\
0.155\end{array}$ & $\begin{array}{l}0.000 * \\
0.249\end{array}$ \\
\hline
\end{tabular}

*: Significant at 5\% level, **: Slopes, ***: Standard errors, ****: P-values.

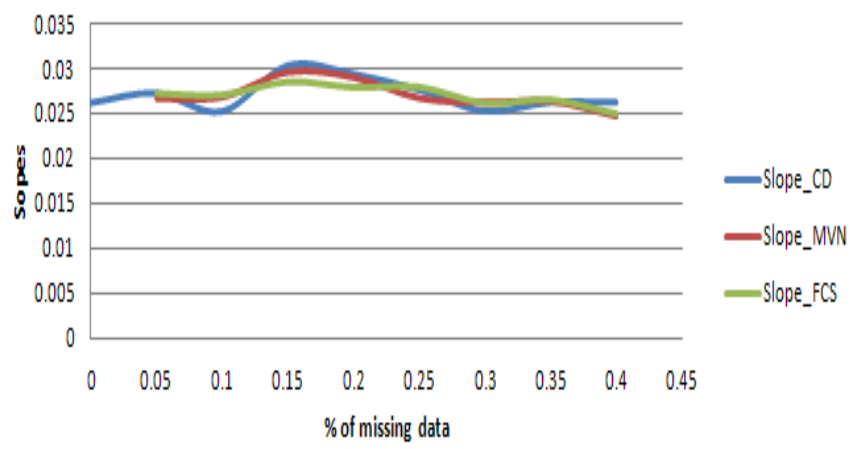

Figure 1. Estimates of slopes for age when the case deletion (CD), multivariate normal imputation (MVN) and fully conditional specification (FCS) methods are used at different rates of missingness. 


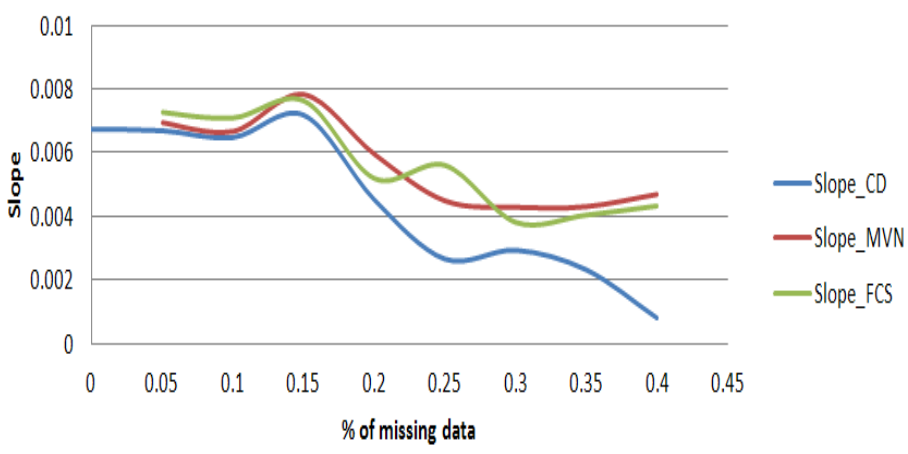

Figure 2. Estimates of slopes for education when the case deletion (CD), multivariate normal imputation (MVN) and fully conditional specification (FCS) methods are used at different rates of missingness.

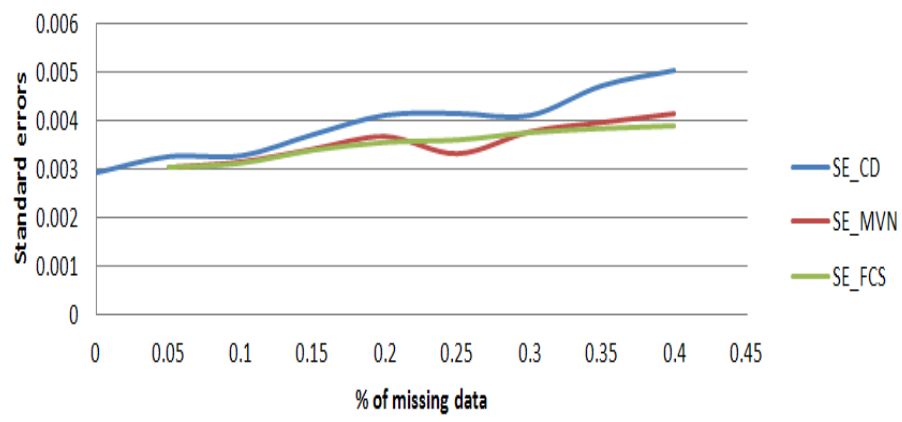

Figure 3. Estimates of standard errors for age when the case deletion (CD), multivariate normal imputation (MVN) and fully conditional specification (FCS) methods are used at different rates of missingness.

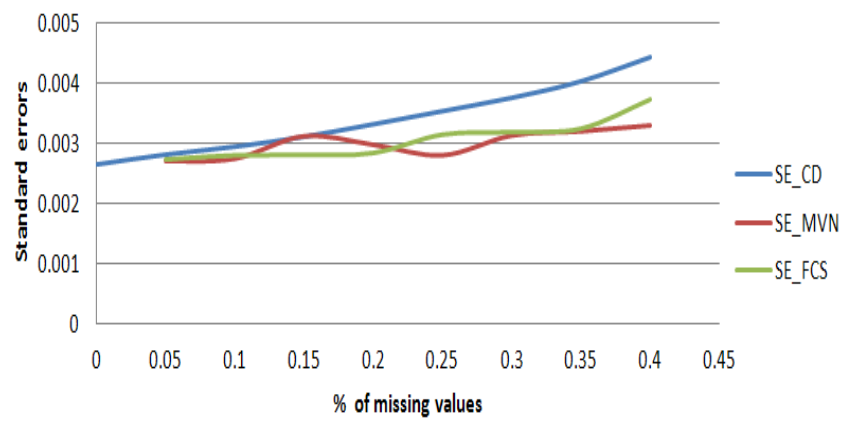

Figure 4. Estimates of standard errors for education when the case deletion (CD), multivariate normal imputation (MVN) and fully conditional specification (FCS) methods are used at different rates of missingness.

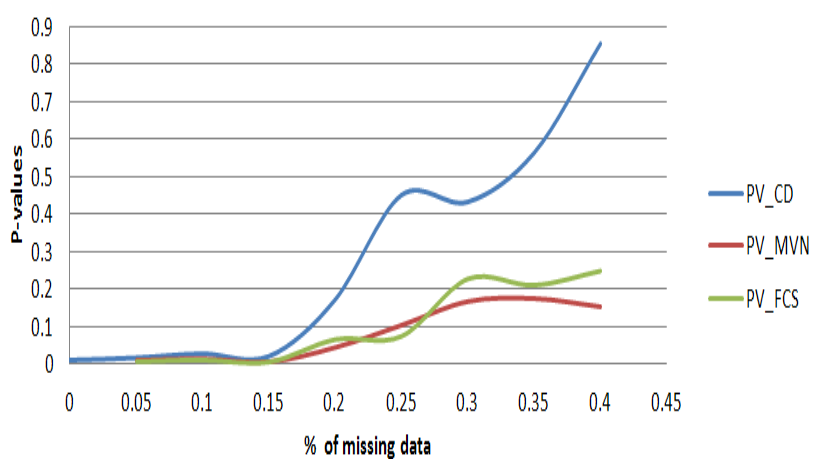

Figure 5. P-values for education when the case deletion (CD), multivariate normal imputation (MVN) and fully conditional specification (FCS) methods are used at different rates of missingness.

Note that these results did not consider other missingness mechanisms namely the missing at random (MAR) and missing not at random (MNAR) mechanisms. The former states that missingness is related to at least one variable in the data set and the latter means that a value of an unobserved variable itself predicts missingness. Secondly, only independent and normally distributed continuous variables were considered in the analysis. Since survey data contains a 
mixture of continuous and categorical variables, a study that looks at the performance of these approaches on categorical independent variables that do not assume normality is also needed.

\section{Conclusion and Limitations}

The MCMC is an iterative procedure used to estimate parameters of interest under difficult conditions such as missing data or when underlying distributions do not fit the assumptions of the maximum likelihood. Its role is to find a probability distribution that can be used to estimate parameters of interest. This paper discussed the mathematics involved in the data augmentation method based on the multivariate normal model (MVN) that uses the MCMC process. A real dataset (the 2007 DRC DHS) was used to compare inferences made using this method (MVN), the case deletion (CD) missing data technique that discards subjects with missing values from the analysis and the fully conditional specification (FCS) multiple imputation method that uses a sequence of regression models to fill in missing values. The performance of these methods under the assumption that data are missing completely at random (MCAR) was highlighted. The results indicated that the higher the proportion of missing data, the more the relationship between variables is distorted when missing data techniques (CD, MVN and FCS) are used. As expected, it was also shown that model-based imputation methods (MVN and FCS) yield less biased estimates than the CD method. Furthermore, the findings indicated that the MVN and the FCS produce similar parameter estimates (van Buuren, 2007) but the MVN is better in terms of preserving an existing relationship between variables at higher rates of missing values. Finally, it was highlighted that at some stage (when the proportion of missing data becomes high, neither the imputation methods nor the case deletion can help to maintain the existing relationship between variables when the analysis is done on the data set with no missing values.

\section{References}

[1] Demirtas, H., Freels, S.A. and Yucel, R.M. (2008). Plausibility of multivariate normality assumption when multiply imputing non-Gaussian continuous outcomes: a simulation assessment. Journal of Statistical Computation and Simulation, 78(1): 69-84.

[2] Enders, C. K. (2010). "Applied Missing Data Analysis", 1st ed. Guildford Press, New York.

[3] Enders, C.K. (2006). A primer on the use of modern missing data methods in psychosomatic medicine research, Psycho- somatic Medicine, 68(3): 427-437.

[4] Galati, J. C. and Carlin, J. B. (2008). INORM: Stata Module to Perform Multiple Imputation Using Schafer's Method [software]. Chestnut Hill, MA: Department of Economics, Boston College, USA.

[5] Gelman, A., Carlin, J.B., Stern, H.S. and Rubin, D.B. (2004). "Bayesian data analysis", second edition. Boca Raton, Chapman \& Hall.

[6] Graham, J.W. (2009). Missing Data Analysis: Making It Work in the Real World, Annual review of psychology, 60: 549-576.

[7] Horton, N.J. and Lipsitz, S.R. (2001). Multiple Imputation in Practice: Comparison of Software Packages for Regression Models With Missing Variables, American Statistical Association, 55: 244-254.

[8] Jackman, S. (2000). Estimation and Inference via Bayesian Simulation: An Introduction to Markov Chain Monte Carlo, American Journal of Political Science, 44: 375-404.

[9] Janssen, K.J.M. (2010). Missing covariate data in medical research: To impute is better than to ignore. Journal of clinical epidemiology, 63: 721-727.

[10] Lee, K.J. and Carlin, J.B. (2010). Multiple Imputation for Missing Data: Fully Conditional Specification Versus Multivariate Normal Imputation. American Journal of Epidemiology, 171(5).

[11] Leeuw, E.D. and Huisman, J. Hox, M. (2003). Prevention and treatment of item nonresponse, Journal of Official Statistics, 19: 153-176.

[12] Little, R. and Rubin, D. (2002). "Statistical Analysis with Missing Data". John Wiley and Sons Inc, New York.

[13] McKnight, P.E. and McKnight, K.M., Sidani, S. and Figueredo, A.J. (2007). "Missing Data: A Gentle Introduction". Guilford Press, New York.

[14] Schafer, J.L. (1997). "Analysis of Incomplete Multivariate Data". Chapman and Hall, London.

[15] Schafer, J.L. and Graham, J.W. (2002). Missing Data: Our View of the State of the Art. Psychological methods, 7(2): 147-177.

[16] Tsikriktsis, N. (2005). A review of techniques for treating missing data in OM survey research. Journal of Operations Management, 24: 53-62.

[17] [van Buuren, S. (2007). Multiple of discrete and continuous data by fully conditional specification. Statistical Methods in Medical Research, 16(3): 219-242.

[18] van Buuren, S. and Knook, D.L. (1999). Multiple Imputation of Missing Blood Pressure Covariates in Survival Analysis. Statistics in Medicine, 18: 681-694.

[19] Yu, L.M. and Burton, A. and Rivero-Arias, O. (2007). Evaluation of software for multiple of semi-continuous data. Statistical Methods in Medical Research: 16(3): 243-258. 